\title{
Spectral dynamics of modulation instability described using Akhmediev breather theory
}

\author{
K. Hammani, ${ }^{1}$ B. Wetzel, ${ }^{2}$ B. Kibler, ${ }^{1, *}$ J. Fatome, ${ }^{1}$ C. Finot, ${ }^{1}$ G. Millot, ${ }^{1}$ N. Akhmediev, ${ }^{3}$ and J. M. Dudley ${ }^{2}$ \\ ${ }^{1}$ Laboratoire Interdisciplinaire Carnot de Bourgogne, CNRS-Université de Bourgogne UMR 5209, 21078 Dijon, France \\ ${ }^{2}$ Institut FEMTO-ST, CNRS-Université de Franche-Comté UMR 6174, 25030 Besançon, France \\ ${ }^{3}$ Optical Sciences Group, Research School of Physics and Engineering, Institute of Advanced Studies, \\ The Australian National University, Canberra ACT 0200, Australia \\ *Corresponding author: bertrand.kibler@u-bourgogne.fr
}

Received March 16, 2011; revised April 28, 2011; accepted May 5, 2011; posted May 10, 2011 (Doc. ID 144328); published June 1, 2011

\begin{abstract}
The Akhmediev breather formalism of modulation instability is extended to describe the spectral dynamics of induced multiple sideband generation from a modulated continuous wave field. Exact theoretical results describing the frequency domain evolution are compared with experiments performed using single mode fiber around $1550 \mathrm{~nm}$. The spectral theory is shown to reproduce the depletion dynamics of an injected modulated continuous wave pump and to describe the Fermi-Pasta-Ulam recurrence and recovery towards the initial state. Realistic simulations including higher-order dispersion, loss, and Raman scattering are used to identify that the primary physical factors that preclude perfect recurrence are related to imperfect initial conditions. (c) 2011 Optical Society of America OCIS codes: $\quad 060.4370,060.5530$.
\end{abstract}

A central process of nonlinear physics is modulation instability (MI), where periodic perturbations on a plane wave are amplified to generate a parametric cascade of spectral sidebands $[1,2]$. During the initial evolution, the sidebands experience exponential growth at the expense of the pump, but the subsequent dynamics are more complex and different scenarios of energy exchange between the spectral modes are possible. This dynamics are closely related to the universal Fermi-Pasta-Ulam (FPU) recurrence phenomenon [3]].

In nonlinear fiber optics, MI has been the subject of extensive study using both numerical simulations and analytical approaches based on truncated sideband solutions to the nonlinear Schrödinger equation (NLSE) [뜨, $\underline{5}$ ]. However, it has recently been shown that MI can be accurately described by exact analytic solutions known as Akhmediev breathers (ABs) [6], and this has led to the design of experiments exciting the rational Peregrine soliton [ $\left[{ }^{2}, 8\right]$. An additional feature of $\mathrm{AB}$ theory is that it allows an analytical description of the spectral decay of the sideband modes at the peak of the MI gain, and this has provided new insights into the development of noise-driven supercontinuum generation $[9,10]$.

In this Letter, we complete the analytic description of $\mathrm{AB}$ spectral dynamics for the case of arbitrary gain, allowing the development of sideband generation in induced MI to be compared with theory under general conditions. Experiments measuring pump and multiple sideband generation over a growth-return cycle of MI are used to quantitatively test the theoretical results. In fact, with the excellent signal to noise ratio in our experiments, we can compare experiment and theory out to more than 10 spectral sidebands and over a $30 \mathrm{~dB}$ dynamic range. To our knowledge, this is the most complete and highest signal of noise characterization of MI dynamics in any NLSE-governed system, representing a significant advance relative to previous studies in optics $[\underline{3}, \underline{11}, \underline{12}]$ and in other systems $[\underline{13}-\underline{15}]$.
Our analysis is based on the normalized NLSE $\psi_{\xi}+1 / 2 \beta_{2} \psi_{\tau \tau}+|\psi|^{2} \psi=0$, where $\psi(\xi, \tau)$ is the field envelope. The $\mathrm{AB}$ solution describes growth and decay of a harmonically perturbed plane wave, and its temporal description in terms of localized compressed pulses is now well-known [6,7]. In addition to the time-domain description, expansion in a Fourier series and appropriate integration yields exact solutions for the pump and spectral harmonic amplitudes. Labeling the amplitudes of the pump and the $n$th sideband, $A_{0}$ and $A_{n}$, respectively, $(n=$ $\pm 1, \pm 2$, etc.), and ignoring factors of constant amplitude and phase, we derive the following results describing pump and sideband evolution with distance:

$$
\begin{aligned}
A_{0}(\xi)= & 1-\frac{i b \sinh b \xi+p^{2} \cosh b \xi}{\sqrt{\cosh ^{2} b \xi-2 a}}, \\
A_{n}(\xi)= & \frac{i b \sinh b \xi+p^{2} \cosh b \xi}{\sqrt{\cosh ^{2} b \xi-2 a}} \\
& \times\left[\frac{\cosh b \xi-\sqrt{\cosh ^{2} b \xi-2 a}}{\sqrt{2 a}}\right]^{|n|} .
\end{aligned}
$$

The parameters $a$ and $b$ are the normalized perturbation frequency and instability growth rate, and in dimensional units (for dispersion $\beta_{2}$ and nonlinearity $\gamma$ ) we have $2 a=$ $\left[1-\left(\omega_{\text {mod }} / \omega_{c}\right)^{2}\right]$ and $b=\left[8 a(1-2 a]^{1 / 2}\right.$. Here, $\omega_{\text {mod }}$ is the dimensional modulation frequency in the range $0<\omega_{\text {mod }}$ $<\omega_{c}$, where $\omega_{c}^{2}=4 \gamma P_{0} /\left|\beta_{2}\right|$ for an initial plane wave power $P_{0}$. The parameter is $p=2^{1 / 2} \omega_{\text {mod }} / \omega_{c}$. The normalized distance is related to dimensional distance $z$ via $\xi=z / L_{\mathrm{NL}}$ with the nonlinear length $L_{\mathrm{NL}}=\left(\gamma P_{0}\right)^{-1}$. We stress that this general result exactly predicts the dynamics of an arbitrary number of sidebands without

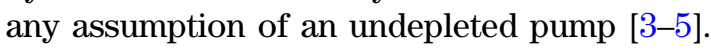




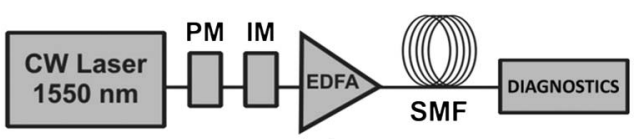

(a)

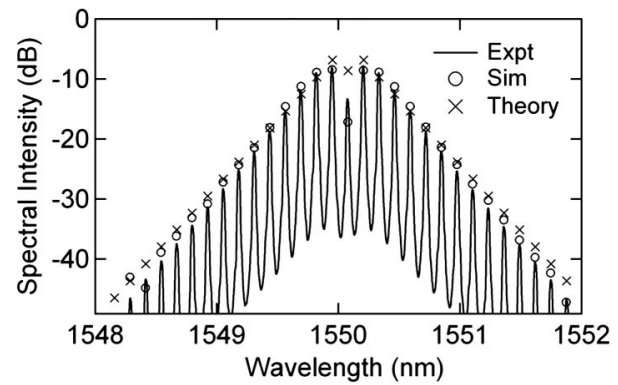

(b)

Fig. 1. (a) Experimental setup. Diagnostics recorded power and spectra. (b) Solid curve, measured spectrum at maximum sideband generation; circles and crosses, simulation and analytic results.

The predictions of Eq. (1) have been compared with simulations and experiments by considering induced MI in standard fiber to be around $1550 \mathrm{~nm}$. Figure 1(a) shows our experimental setup. A laser diode at $155 \overline{0.08}$ $\mathrm{nm}$ passes through a $16 \mathrm{GHz}$ intensity modulator (IM) to generate two equal amplitude sidebands at $-15.6 \mathrm{~dB}$ relative to the pump. This weak modulation is that where the $\mathrm{AB}$ theory is expected to well-describe the propagation dynamics, and a phase modulator (PM) is used for linewidth broadening to suppress Brillouin scattering. The power after modulation was adjusted using a low noise erbium-doped fiber amplifier (EDFA). The use of a standard single mode fiber (SMF-28) in our experiments allows fusion splicing to be performed with negligible loss so that we could readily study the spectral evolution dynamics using cutback experiments and so that we could also repeat experiments for a range of different conditions. The fiber parameters were $\beta_{2}=-21.4 \mathrm{ps}^{2} \mathrm{~km}^{-1}, \beta_{3}=0.012 \mathrm{ps}^{3} \mathrm{~km}^{-1}$, and $\gamma=$ $1.2 \mathrm{~W}^{-1} \mathrm{~km}^{-1}$, and the linear loss was $0.19 \mathrm{~dB} \mathrm{~km}^{-1}$. Here, $P_{0}=0.46 \mathrm{~W}$ so that the normalized modulation frequency $a=0.45$, such that we are far from the MI gain peak and we perform a valid test of the generalized $\mathrm{AB}$ spectral result in Eq. (1).

Figure 1(b) shows typical results using a fiber length of $5 \mathrm{~km}$ and with the input power adjusted to generate a maximally broadened spectrum. With the low noise levels in our experiment, we could resolve more than 14 sidebands on either side of the pump (solid curve). The experimental results are compared with the predictions of Eq. (1) (the crosses) and we see very good agreement in predicting spectral decay over more than 4 orders of magnitude. The figure also shows results of numerical simulations which are also in good agreement. The simulations used an extended NLSE, including third-order dispersion and Raman scattering, but we found that these additional effects have little effect at the point of maximum spectral broadening.

For the same parameters as above $(16 \mathrm{GHz}$ and $a=$ 0.45 ), we then performed experiments to examine the evolution dynamics more fully. Specifically, the fiber length was changed over the range $0-8.5 \mathrm{~km}$ and spectral
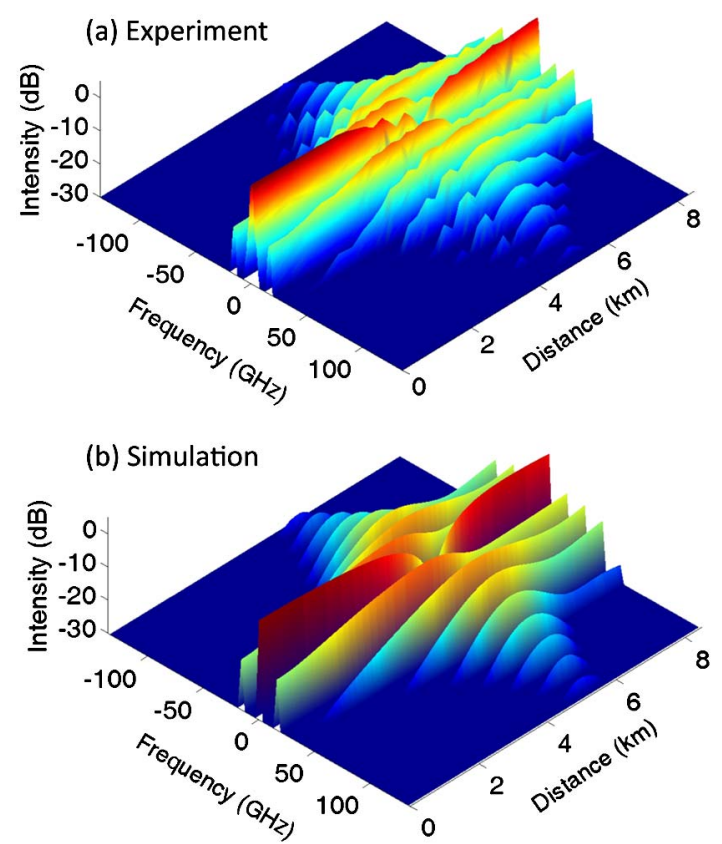

Fig. 2. (Color online) Dynamics of multiple sideband generation. (a) Experimental results. (b) Results from numerical simulation using realistic NLSE model. The generation of multiple sidebands around $5 \mathrm{~km}$ and recovery back towards the initial state with further propagation is clearly seen.

measurements were made at $\sim 250 \mathrm{~m}$ intervals. The distance of $8.5 \mathrm{~km}$ was chosen to provide detailed study of the first growth-decay cycle of AB evolution. Figure 2(a) plots these measurements. For visual clarity, we plot only nine sidebands and use a plotting floor at $-30 \mathrm{~dB}$, although the dynamic range of our measurements extended further as discussed above and shown in Fig. 1(b).

These results are significant in clearly showing the full instability dynamics of multiple sideband generation, as well as the recovery back towards the initial state with propagation beyond $5 \mathrm{~km}$. The experimental results in Fig. 2(a) were compared with both numerical simulations and the theoretical predictions from the spectral $\mathrm{AB}$ theory in Eq. (1). The simulation results (again using an extended NLSE) explicitly show that although propagation beyond $5 \mathrm{~km}$ sees the expected spectral compression as the sideband energy begins to be converted back to the pump, ideal FPU recurrence is not observed. Rather, after around $7 \mathrm{~km}$, we see the onset of more complex dynamics. In this context, we note that we have been able to perform simulations with and without the higher-order terms in the NLSE, and this has allowed us to clarify that loss, third-order dispersion, and the Raman effect in fact have no influence on this deviation from ideal propagation behavior. Rather, it is the nonideal initial conditions (which do not correspond to the mathematical ideal case for a single $A B$ cycle) that induce the deviation from recovery to the initial state.

A full quantitative comparison between the experiment and the predictions of Eq. (1) is shown in Fig. 3. Here, we extract the experimental pump and sideband intensity for various sideband orders $n$ (circles), and compare the evolution as a function of distance with corresponding results from the simulations (blue short dashes) and the $\mathrm{AB}$ spectral theory (black solid curve). The spectral 

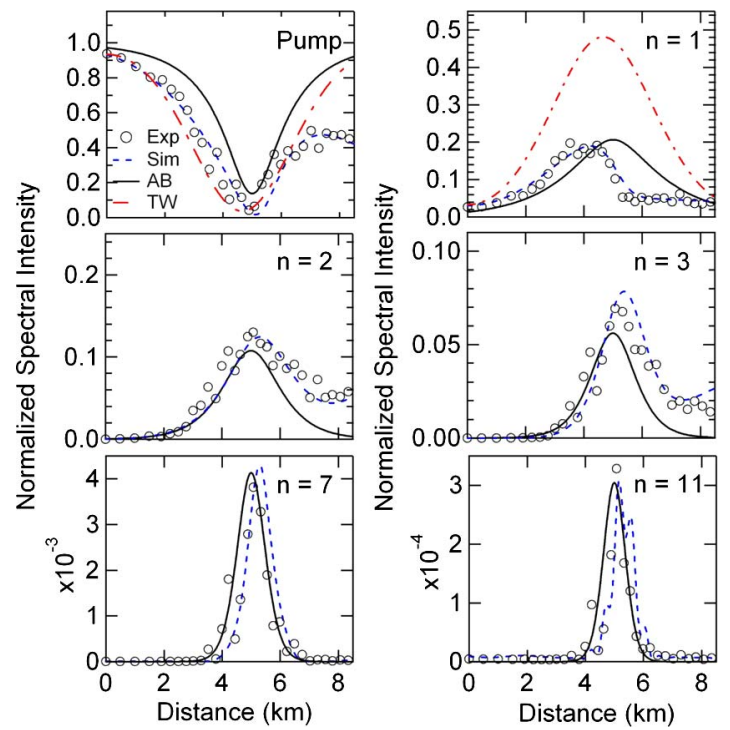

Fig. 3. (Color online) Spectral dynamics for the pump and sideband orders as shown. Experiment (circles) is compared with simulation (blue dashed curve), the exact $\mathrm{AB}$ result Eq. (1) (black curve), and the truncated TW model (red dotdashed curve).

normalization in all cases is relative to the total initial energy. In addition, for completeness, for the pump and $n=$ 1 sideband, the red long dashes show the predictions of the well-known truncated three wave (TW) model [4, $\underline{5}$ ].

It is important to note that no free parameters were used in the comparison between experiment and simulation or the TW theory. Also, only the distance offset for the $\mathrm{AB}$ theoretical curve was adjusted to match the position of maximum pump depletion; once this was fixed, no relative normalization of pump or sideband intensity to experimental or simulation data was made. The agreement between simulation and experiment is very good, but this of course might be expected given the fact that the simulation's initial conditions were chosen to match exactly those used in the experiment. Indeed, we have checked in other experiments that the simulation agreement remains excellent to longer distances beyond the first cycle of growth-decay. We also note that the TW theory accurately predicts the distance to maximum compression and the extent of pump depletion. However, because it assumes that all the depleted energy is transferred only to the first sideband pair, the TW theory significantly overestimates the sideband amplitude.

What is particularly impressive is the degree to which the $\mathrm{AB}$ theory describes both the dynamical evolution and the quantitative extent of pump depletion and sideband growth, even out to the $n=11$ sideband. In fact, our ability to study dynamics to such a high sideband order reveals a previously uncommented feature of the dynamics where we see that the temporal compression is associated with an increased spatial localization of the energy transfer to higher sideband orders.

Although the AB theory has been previously shown to describe field characteristics at the maximal spectral broadening, these results now confirm its validity in describing the dynamical evolution from growth to decay over an MI cycle. Realistic simulations using an extended NLSE identify that the primary physical factors that preclude perfect recurrence are due to imperfect initial conditions, specifically a nonzero relative phase between injected pump and sidebands. With suitable additional control of the injected field, we anticipate that creation of ideal nonlinear breather states should be possible. These results show how analytical breather solutions of the NLSE provide new insights into seemingly wellknown processes such as fiber MI.

We thank the French Agence Nationale de la Recherche projects MANUREVA ANR-08-SYSC-019 and IMFINI ANR-09-BLAN-0065, the Conseil Régional de Bourgogne, and the Australian Research Council Discovery Project DP110102068 for support.

\section{References}

1. T. B. Benjamin and J. E. Feir, J. Fluid Mech. 27, 417 (1967).

2. V. I. Bespalov and V. I. Talanov, JETP Lett. 3, 307 (1966).

3. G. Van Simaeys, P. Emplit, and M. Haelterman, Phys. Rev. Lett. 87, 033902 (2001).

4. G. Cappellini and S. Trillo, J. Opt. Soc. Am. B 8, 824 (1991).

5. S. Trillo, S. Wabnitz, and T. A. B. Kennedy, Phys. Rev. A 50, 1732 (1994).

6. N. Akhmediev and V. I. Korneev, Theor. Math. Phys. 69, 1089 (1986).

7. B. Kibler, J. Fatome, C. Finot, G. Millot, F. Dias, G. Genty, N. Akhmediev, and J. M. Dudley, Nature Phys. 6, 790 (2010).

8. K. Hammani, B. Kibler, C. Finot, P. Morin, J. Fatome, J. M. Dudley, and G. Millot, Opt. Lett. 36, 112 (2011).

9. J. M. Dudley, G. Genty, F. Dias, B. Kibler, and N. Akhmediev, Opt. Express 17, 21497 (2009).

10. N. Akhmediev, A. Ankiewicz, J. M. Soto-Crespo, and J. M. Dudley, Phys. Lett. A 375, 775 (2011).

11. D. L. Hart, A. Judy, R. Roy, and J. W. Beletic, Phys. Rev. E 57, 4757 (1998).

12. J. Beeckman, X. Hutsebaut, M. Haelterman, and K. Neyts, Opt. Express 15, 11185 (2007).

13. M. P. Tulin and T. Waseda, J. Fluid Mech. 378, 197 (1999).

14. N. Karjanto and E. van Groesen, J. Hydro-Environ. Res. 3, 186 (2010).

15. M. M. Scott, B. A. Kalinikos, and C. E. Patton, J. Appl. Phys. 94, 5877 (2003). 